\title{
Retropharyngeal tuberculosis abscess: A case report and review of the literature
}

\author{
Bilge Turk*, Meltem Akpınar, Kerem Sami Kaya and Suat Turgut \\ Department of Otorhinolaryngology Head and Neck Surgery, Şişli Hamidiye Etfal Training and Research Hospital, Turkey
}

\begin{abstract}
Objective: Retropharyngeal tuberculosis abscess is a rare presentation of extra pulmonary tuberculosis and a potentially life-threatening deep neck infection. The diagnosis and early management of the disease is important and delays may result in severe disabilities and poor outcome.

Case report: A 30 years-old male patient was referred to outpatient clinic with complaints of dyphagia and difficulty on swallowing. Symptoms or signs of tuberculosis was not present. Physical examination and computed tomography of neck revealed retropharyngeal abscess. Despite intraoral surgical drainage and antituberculosis medical treatment, patient complaint upper limp muscle weakness. After cervical exposure and drain placement into the abscess, his dysphagia and upper limp muscle weakness symptoms gradually improved.
\end{abstract}

Conclusion: Extra pulmonary tuberculosis should be dealt with high degree suspicion in case of abcesses refractory to medical treatment and surgical drainage especially in endemic countries in order to reduce disabilities and improve clinical outcome.

\section{Introduction}

The incidence of extrapulmonary tuberculosis is rare and mainly affects theyoung and middle-aged men along with immunocompromised individuals [1]. One of the clinical presentation of extrapulmonary tuberculosis is retropharyngeal abscess of tuberculosis that is a potentially life-threatening deep neck infection [2].

The retropharyngeal space communicates bilaterally with the Para pharyngeal spaces and posteriorly with danger space. Due to that close interaction, infections of the retropharyngeal space can be life threatening. Extramedullary intradural tuberculosis is a rare entity with few cases described in the world literature, and the history of tuberculous meningitis or anti-tuberculosis treatment is usually present in the patients. Lymph nodes, pleura, genitourinary tract, bones and joints, the meninges, peritoneum and pericardium are the common sites of extrapulmonary tuberculosis in descending order of frequency. A case with retropharyngeal tuberculosis abscess with recurrence despite surgical drainage and intense medical treatment is reported.

\section{Case presentation}

A 30 years-old male patient was admitted with the complaint of progressive dysphagia. His past medical history was unremarkable. The patient has no fever and did not mention any night sweating.

Physical examination revealed swelling of the posterior wall of the oropharynx with no imminent airway compromise. Upon neck palpation, multiple bilateral lymphadenopathies were detected at level 2 and 3. Flexible endoscopy of the vocal cords, auscultation of the lungs were normal. Laboratory studies showed elevated inflammatory parameters including a white blood cell count of 13660 /uL, ESR of 84 $\mathrm{mm}$, CRP $101.06 \mathrm{mg} / \mathrm{L}$. Patient is immunocompetent with negative viral markers for human immunodeficiency virus (HIV) and Ebstein Barr virus (EBV). Chest X-ray did not show pulmonary infiltration.
Computed tomography (CT) scan of neck showed an $84 \times 38 \times 94$ $\mathrm{mm}$ retropharyngeal abscess extending superiorly to nasopharyngeal level, inferiorly to the mediastinum, and posteriorly to the prevertebral space (Figure 1).

Magnetic resonance (MR) imaging revealed a large retropharyngeal and epidural abscess with involvement of pre-cervical soft tissues. Signal difference was detected at the cervical vertebral corpuses. The abscess extended to deep neck muscles and through the vertebral foramens to the anterior part of the cervical spinal canal (Figure 2).

Transcervical aspiration of the abscess was applied and $150 \mathrm{ml}$ of pus was aspirated. The pus was examined for microbiology and cytology. There was a subjective improvement of symptoms after aspiration. Ampiric antibiotic treatment was prescribed. The bacteriological examination revealed acid fast stained bacillus in the pus aspirated from the abscess. Owing to the high clinical likelihood of tuberculosis, empiric antibiotic regimen was stopped, and anti-tuberculosis regime was promptly initiated. But no significant improvement was observed. The patient had upper limb weakness while on medical treatment. The physical examination revealed muscle wasting with a loss of power on a scale of 3/5 in upper limbs.

Upon lack of improvement in clinical symptoms and radiological findings, intraoral surgical drainage was performed in reverse trendelenburg position under general anesthesia. An incision to the posterior wall of the oropharynx was done and plenty amount of pus was aspirated. In order to avoid the spread of contents, special care

*Correspondence to: BilgeTurk, Department of Otorhinolaryngology Head and Neck Surgery, Şişli Hamidiye Etfal Training and Research Hospital, Istanbul, Turkey, Tel: +90 532 2868213, E-mail: drbilgeturk@hotmail.com

Key words: retropharyngeal abscess, tuberculosis, extrapulmonary tuberculosis

Received: June 06, 2018; Accepted: June 22, 2018; Published: June 27, 2018 
was taken. In early postoperative period, there was no complication. Despite the regular four agent anti-tuberculosis treatment (isoniazid, rifampicin, ethambutol and pyrazinamide orally) and intraoral drainage, the patient had symptoms of paresthesia in the upper limbs and his complaints continued to deteriorate. Neck and brain MRI was performed on postoperative 7 th day and revealed that the size of retropharyngeal abscess declined but still collection was present and epidural abscess was also existing. An open surgery via cervical approach was planned. An incision was made medial to the carotid bifurcation level and posterior to the pharyngeal space at the right side. The pus was aspirated, and the cavity was washed with serum physiologic solution. A penrose drain was placed for 6 days. During the postoperative course, a control CT scan revealed the regression of the abscess and the drain in the cavity was in place (Figure 3).

The drain was taken out on the postoperative $6^{\text {th }}$ day, scar healed well, and postoperative period was uneventful. The muscle weakness improved gradually after the drainage. The patient was discharged on the $10^{\text {th }}$ postoperative day and still on course of four agent anti-

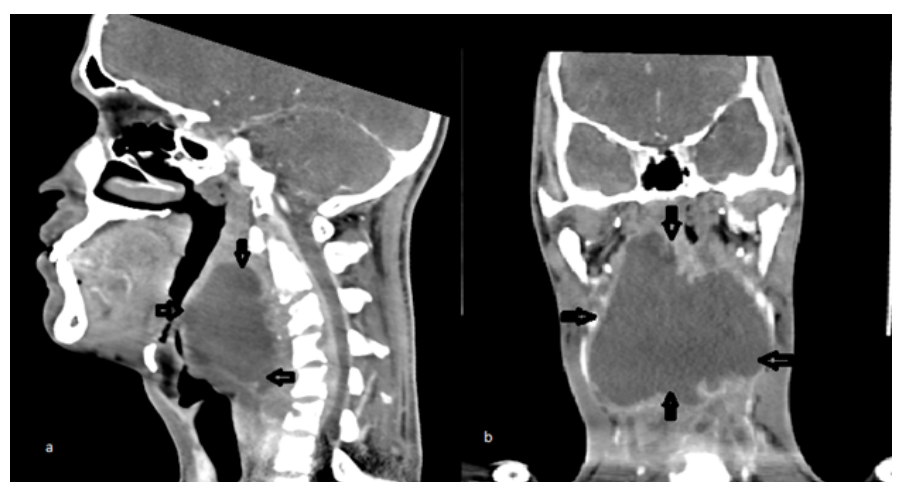

Figure 1. Neck computed tomography. (a) Sagittal view of the retropharyngeal abscess extending superiorly to nasopharyngeal level, inferiorly to the mediastinum, posteriorly to the prevertebral space; (b) Coronal view of the retropharyngeal abscess

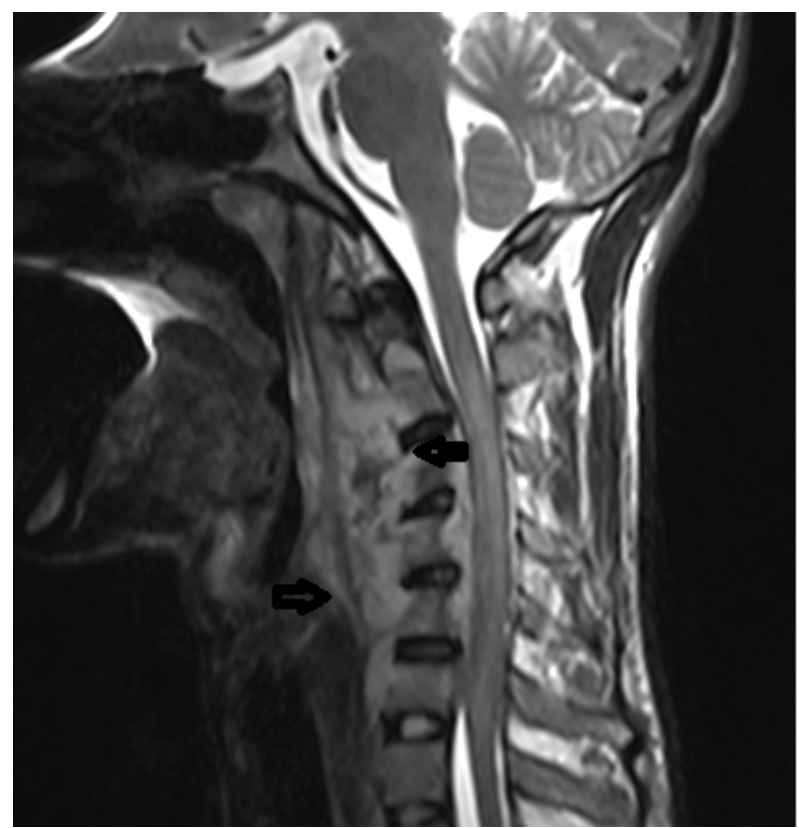

Figure 2. Sagittal magnetic resonance imaging of the neck reveals large retropharyngeal and epidural abscess with involvement of pre-cervical soft tissues. Signal difference was detected at the cervical vertebral corpuses

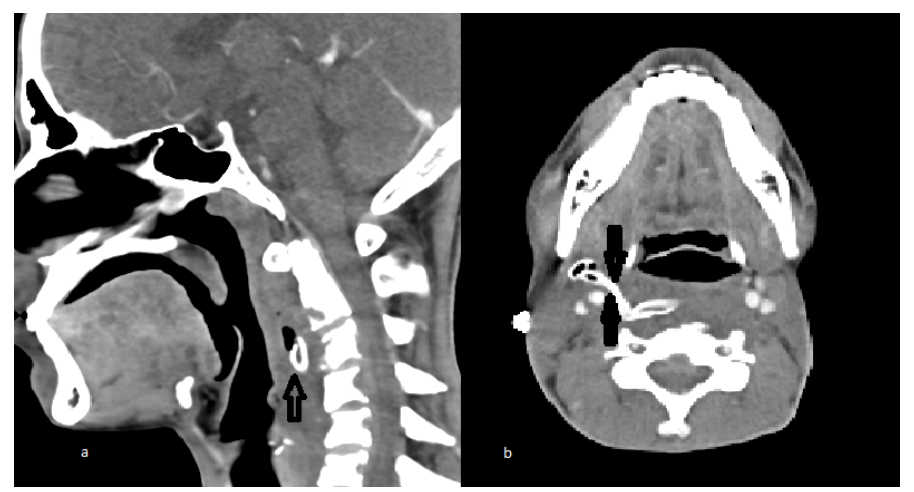

Figure 3. Postoperative neck computed tomography findings. (a) sagittal and (b) axial view of the retropharyngeal abscess regressed after the surgical drainage, arrow showing the drain placed in the abscess

tuberculosis treatment. Patient was informed to watch for complications of the disease and anti-tuberculosis drugs.

\section{Discussion and conclusion}

Tuberculosis infection is an entity of high incidence in developing countries and commonly involves the lungs but can also affect other parts of the body. Most common presentation of extrapulmonary tuberculosis is cervical lymphadenitis as a single or multiple painless swelling [3]. Despite of availability of effective anti-tuberculosis drugs, tuberculosis remains leading cause of death among curable infectious diseases.

Tuberculosis retropharyngeal abscess is a rare presentation of extra pulmonary tuberculosis and a potentially life-threatening deep neck infection. The diagnosis is based on careful patient's history and physical examination along with high index of clinical suspicion. The diagnosis of the disease with unusual presentation is difficult but also important since retropharyngeal space continues inferiorly into the mediastinum and delay in diagnosis and treatment may result in severe morbidity and poor outcome.

In order to assess the extent of the disease and possible complications, radiological imaging is important. A computed tomography scan can accurately differentiate cellulitis from an abscess while a magnetic resonance imaging provides a better evaluation of soft tissues in the neck and is useful in assessing the vascular complications [4,5].

Early diagnosis is key to appropriate and definite treatment. However, there is still lack of guidelines on the accurate treatment and management strategy for cervical spine tuberculosis and subsequent retropharyngeal abscess that makes the treatment more complicated and challenging [6]. In fact, it is hard to suspect tuberculosis in the first place, due to the similar and common symptoms of retropharyngeal abscess and common symptoms and signs may be masked. In our patient, there was no classical symptoms of spinal tuberculosis in the beginning and thus the diagnosis was delayed.

For the tuberculosis diagnosis the latter is a highly specific investigative modality, but sensitivity is low. In clinical samples PCR technique is sensitive for detecting mycobacterium and this technique also can provide information regarding drug-resistant strains [7]. Nowadays different tests like interferon- $\gamma$ release assays are available for the diagnosis of tuberculosis but there is little evidence for the use of these tests in the literature [8].

Although surgical drainage improves compressive symptoms, medical therapy remains the mainstay in the treatment of tuberculomas. 
For pulmonary tuberculosis the standard recommended regimen is 6 months of isoniazid and rifampicin, supplemented in the first 2 months with pyrazinamide and ethambutol. However in case of extrapulmonary tuberculosis including bone involvement, the recommended duration of anti-tuberculosis medication is $9-12$ months $[9,10]$. In the present case the duration of medical treatment was planned as 12 months.

Extra pulmonary tuberculosis should be suspected in cases associated with abscesses refractory to medical treatment and surgical drainage especially in endemic countries to reduce disabilities and improve clinical outcome.

\section{References}

1. Prakasha SR, Suresh G, D'sa IP, Shetty SS, Kumar SG (2013) Mapping the pattern and trends of extrapulmonary tuberculosis. J Glob Infect Dis 5: 54-59. Crossref]

2. Tannebaum RD (1996) Adult retropharyngeal abscess: a case report and review of the literature. J Emerg Med 14: 147-158. [Crossref]

3. Maharjan M, Hirachan S, Kafle PK, Bista M, Shrestha S, et al. (2009) Incidence of tuberculosis in enlarged neck nodes, our experience. Kathmandu Univ Med J (KUMJ) 7: 54-58. [Crossref]
4. Miller WD, Furst IM, Sandor GK, Keller MA (1999) A prospective, blinded comparison of clinical examination and computed tomography in deep neck infections. Laryngoscope 109: 1873-1879. [Crossref]

5. Gidley PW, Ghorayeb BY, Stiernberg CM (1997) Contemporary management of deep neck space infections. Otolaryngol Head Neck Surg 116: 16-22. [Crossref]

6. Schuler PJ, Cohnen M, Greve J, Plettenberg C, Chereath J, et al. (2009) Surgical management of retropharyngeal abscesses. Acta Otolaryngol 129: 1274-1279. [Crossref]

7. Xu HB, Jiang RH, Sha W, Li L, Xiao HP (2010) PCR-single-strand conformational polymorhism method for rapid detection of rifampin-resistant mycobacterium tuberculosis: systematic review and meta-analysis. J Clin Microbiol 48: 3635-3640. [Crossref]

8. Denkinger CM, Dheda K, Pai M (2011) Guidelines on interferon-? release assays for tuberculosis infection: concordance, discordance or confusion? Clin Microbiol Infec 17: 806-814. [Crossref]

9. Lange C, Abubakar I, Alffenaar JWC, Bothamley G, Caminero JA, et al. (2014) Management of patients with multidrug-resitant/extensively drug-resistant tuberculosis in Europe: a TBNET consensus statement. Eur Respir J 44: 23-63. [Crossref]

10. Jain AK (2010) Tuberculosis of the spine: a fresh look at an old disease. J Bone Joint Surg $\operatorname{Br}$ 92: 905-913. [Crossref]

Copyright: (C2018 Turk B. This is an open-access article distributed under the terms of the Creative Commons Attribution License, which permits unrestricted use, distribution, and reproduction in any medium, provided the original author and source are credited. 